Durch die Verleihung des Nobelpreises für Medizin an

\section{Frank Macfarlane Burnet}

(geb. 1899; Professor für Experimentalmedizin an der Universität Melbourne (Australien) und Direktor des "Walter and Eliza Hall Institute for Medical Research" in Melbourne; Spezialist auf den Gebieten der Virologie und Immunbiologie; 1951 in den Ritterstand erhoben; Träger des Lasker-Preises (USA); Mitglied des "Order of merit" $(G B))$ und

\section{Peter Brian Medawar}

(geb. 1915; seit 1951 Professor für Zoologie und vergleichende Anatomie am University College in London; Spezialist auf dem Gebiete der Immunologie und der Zellenumwandlungen) wurden nicht nur zwei hervorragende Forscher geehrt, sondern auch eine vorbildliche $\mathrm{Zu}$ sammenarbeit von medizinischer und zoologischer Forschung und eine neue Disziplin, welche von JoHn M. ConVERSE anlässlich der vierten Gewebehomotransplantationskonferenz im Februar 1960 in New York als "Transplantationsbiologie» charakterisiert wurde.

Durch serologische Untersuchungen wies R. D. OwEN 1945 nach, dass die meisten dizygotischen Rinderzwillinge infolge der synchorialen Gefässanastomosen bei der Geburt Blutchimàren sind. Auf Grund dieser Tatsache postulierten F. M. BurNet und F. FenNer 1949, dass durch den embryonalen Blutaustausch bei den Rinderzwillingen ein Zustand gegenseitiger Verträglichkeit entstehen muss, indem die immunologisch kompetenten Systeme durch den fruhzeitigen Kontakt mit den Antigenen ihrer genetisch verschiedenen Partner spezifisch modifiziert werden, ähnlich wie dies normalerweise gegenüber autologen Gewebeantigenen geschieht. Bereits 1951 und 1952 konnten MEDAWAR et al, zeigen, dass die dizygotischen $Z$ willingskälber gegenseitige Hauttransplantate ebensogut wie genetisch identische Zwillinge tolerieren, während sie Transplantate anderer Geschwistertiere wie solche gewöhnlicher homologer Partner in kurzer Zeit zerstören. In der Folge gelang es P. B. MEDAwar, in Zusammenarbeit mit R. E. Billingham und I. BREnt das Phänomen der erworbenen immunologischen Toleranz durch eine Reihe bewundernswerter Untersuchungen an $\mathbf{K a}$ ninchen, Nagern und Vögeln (vgl. BILlinghaM, BRENT und Medawar 1956 und Medawar 1958) in seiner theoretischen und praktischen Bedeutung klarzulegen. Die Untersuchungen ergaben, dass antigenische Reize, die bei einem adulten Organismus sensibilisierend und Immunität auslösend wirken, bei embryonalen oder neugeborenen Wirtstieren immunologische Toleranz gegen diese Antigene induzieren. Beide Reaktionsphasen sind ontogenetisch durch eine Nullphase miteinander verbunden, die relativ zur Geburt recht verschieden liegen kann. Damit wurde die Theorie von BurNex bestätigt, wonach sich im foetalen und frühkindlichen Leben die immunbiologische Abwehr stufenweise entwickeln soll und sich ein Organismus deswegen vor dem Auftreten einer biologischen Abwehrbereitschaft an artfremdes Gewebe gewöhnen liesse. Die Antigene, welche die Immunreaktion gegen ein Transplantat auslösen, wurden im Zellkern lokalisiert, wobei sich an Lipoide gebundene AminosäurenPolysaccharidkomplexe als wirksam erwiesen (MEDAWAR 1958). Zur Auslösung yon Toleranz sind dagegen lebende kernhaltige Zellen notwendig. Ihre Wirkung ist spender-, aber nicht gewebespezifisch. Bei der Immunisierung gegen ein Transplantat treten zwar auch Serumantikörper auf, doch sind diese in der Regel nicht für die Zerstörung der fremden Gewebe verantwortlich; sie können im Gegenteil zu einer Lebensverlängerung der'Transplantate führen, am deutlichsten bei Tumoren (N. KaIISS 1958). Die wirksamen Antikörper sind zellgebunden, was durch Trans- plantation immunisierter Zellen regionaler Lymphknoten in tolerante isogene Wirte bewiesen wurde, in denen diese Zellen die bisher vom Wirt tolerierten Transplantate zerstörten. Gleichzeitig wurde damit gezeigt, dass auch ein toleriertes Transplantat seine antigenische Wirksamkeit nicht einbüsst. Bringt man immunologisch kompetente (lymphatische oder blutbildende) Zellen eines adulten Spenders in einen toleranten Wirt (Embryo, $F_{1}-$ Hybride, bestrahltes adultes Tier), so werden sie dort gegen die Antigene des Wirts sensibilisiert, verursachen dessen Erkrankung ("homologous disease") und, direkt oder indirekt, dessen baldigen Tod. Damit sind auch die Möglichkeiten und Gefahren aufgezeigt, die sich bei der Gewebetransplantation zwischen nicht verwandten Menschen ergeben, besonders nach Injektion blutbildender Zellen nach Totalbestrahlung. Entscheidende Beiträge liefern die Untersuchungen iiber die erworbene immunologische Toleranz ferner für das Verständnis der immunologischen Beziehungen zwischen Mutter und Fetus und ihrer klinischen Komplikationen, der Tuberkulinallergie und der Autoimmunreaktion (H. S. LAWRENCE 1959), der Zellphänotypen (J: SchulTz 1959) und der Antikörperbildung allgemein (F. M. Burner 1956).

G. ANDRES

\section{CONGRESSUS}

\section{Canada \\ VIII International Congress for Microbiology \\ Montreal, August 19-25, 1962}

The VIII International Congress for Microbiology will be held at Montreal (Canada), from August 19 to 25, 1962, under the auspices of the Canadian Society. of Microbiologists. Headquaters of the Congress will be at new Queen Elizabeth Hotel.

There will be five Sections: Structure and Function; Agricultural Microbiology; Industrial Microbiology; Medical and Veterinary Microbiology; and Virology.

Enquiries should be made to Dr. N. E. GibBons, VIII International Congress for Microbiology, National Research Council, Ottawa 2 (Canada), Requests to be placed on the mailing list for the Second Circular should be made before January 31, 1961.

\section{CORRIGENDUM}

H. KAPPELER und R. SCHWYZER: Synthetic Peptides Related to the Corticotropins (ACTH) and the Melanophore Stimulating Hormones (MSH) Possessing Corticotropin Realeasing Activity (CRF)-Activity). Exper. vol. XVI, fasc. 9, p. 415 (1960).

Infolge eines Irrtums der Druckerei wurden beim Umbruchsatz die Korrekturen der Fussnoten nicht ausgeführt. Korrigierte Separata können beim Verlag angefordert werden.

D. W. Mathieson, B. Jagues, G. T. Chapman, V. P. ARYA, and B. G. ENGEL: The Structure of Cassamine and Erythrophlamine, Exper. XVI, fasc. 9, p. 404 (1960).

The formulae on page 405 are to be corrected as follows: Formula II, position 4: In place of $\mathrm{CCOOH}_{3}$, read $\mathrm{COOH}_{3}$. Formula IX, position 7: In place of $=\mathrm{OH}$, read $=\mathrm{O}$. Formulae IX and $X$, positions 3 and 4: The point of attachement of the hydroxyl group is misprinted: it should be at position 3 and the hydroxyl group at position 4 is to be replaced by a methyl group. 